Type of the Paper (Communication.)

\title{
Jeans Instability, Jeans Entropy and the Entropy Origin of Gravity
}

\author{
Edward Bormashenko
}

Ariel University, Engineering Faculty, Chemical Engineering Department, Ariel, Israel, 407000.

E-mail: edward@ariel.ac.il

*Correspondence: edward@ariel.ac.il

\begin{abstract}
An entropic origin of gravity is re-visited. Isothermal self-gravitating cloud seen as an ideal gas is analyzed. Gravitational attraction within the isothermal cloud in equilibrium is balanced by the pressure, which is of a pure entropic nature. The notion of the Jeans entropy of the cloud corresponding to the entropy of the self-gravitating cloud in mechanical and thermal equilibrium is introduced. Balance of the gravitational compression and the entropic repulsion yields the scaling relation hinting to the entropic origin of the gravitational force. The analysis of the Jeans instability enables elimination of the "holographic screen" or "holographic principle" necessary for grounding of the entropic origin of gravity.
\end{abstract}

Keywords: gravity; entropic force; Jeans instability; isothermal self-gravitating cloud.

\section{Introduction}

The origin and physical nature of gravity was subjected to the stormy scientific discussion recently. The discussion was started by the paper by Eric Verlinde in which Newton's law of gravitation appears within a theory in which space emerges through a holographic scenario [1]. Gravity is identified with an entropic force caused by changes in the information associated with the positions of material bodies [1-2]. The idea was developed by Verlinde in the recent paper in which it was demonstrated that the observed dark energy and the phenomena traditionally attributed to dark matter have a common origin and are connected to the emergent nature of space-time and gravity [3]. The entropic origin of gravity was discussed and developed in refs. 4-5. In particular it was shown, that classical Newtonian gravity may be interpreted in terms of an entropic force; it was also shown that the fact that Newtonian gravity is described by a conservative force places significant constraints on the form of the entropy and temperature functions [4].

The entropy origin of gravity was criticized in refs. 6-8. Kobakhidze argued that experiments with ultra-cold neutrons in the gravitational field of Earth disprove the entropic origin of gravitation [6]. Gao argued, in turn, that neither holographic screen nor test particle satisfies all requirements for the existence of entropic force in a thermodynamics system [7]. Yang suggested that gravity can be seen as entropic force only for systems with constant temperature and zero chemical potential [8].

We conjecture that that the weakest point in the argumentation by Verlinde, resulting in the relating of gravity to entropy forces is the "holographic screen" which seems to be a fanciful intellectual construction, to be eliminated due to the "Occam razor". We demonstrate that the analysis of the Jeans gravitational instability [9-12] supports the entropic origin of the gravity and enables elimination of the "holographic principle". 


\section{Results}

The instability of self-gravitating systems was first studied by Jeans in nonrelativistic Newtonian gravity [9]. It was extended to General Relativity by Evgeny Lifshitz in 1946 and it is broadly used in modern cosmology to study the evolution of density perturbations in the expanding universe [10, 13, 14]. We demonstrate that reinterpretation of the Jeans instability enables new insights in the entropy nature of gravity.

Consider the self-gravitating spherical cloud with the mass of $M$ and radius $r$. The total self-gravitational energy of the cloud, is calculated in a way resembling the calculation of the Born energy of the electrically charged particle (ion) [15]. Imagine the process of gravitational cloud formation by gradually increasing its mass from zero to its full mass $M$. At any stage of this process let the mass of the cloud be $m$ and let this be incremented by $\mathrm{dm}$. The work done in bringing this additional mass, and therefore the total change in the potential energy of the cloud is given by Eq. 1:

$$
\Delta U_{g r}=\int_{0}^{M} G \frac{m d m}{r}=\frac{G M^{2}}{2 r}
$$

where $G$ is the gravitational constant. Again, the resemblence of Eq. 1 to the expression obtained for the Born energy of the charged particle should be emphasized. Jeans instablity emerges in the self-gravitating cloued when perturbations occurring on the sufficiently large spatial scale become unstable, such "large" instablities cause the matter in the cloud to clump together and lead to gravitational collapse [9-14]. The traditional approach to the analysis of the Jeans instablity implies treatment of the sel-gravitating cloud as a continuous liquid. Combining of the Euler equation, continuity equation and the state equation of the self-gravitating liquids yields the following result, the perturbations with $\lambda>\lambda_{J}$ become unstable, where the Jeans length $\lambda_{J}$ is supplied by Eq. 2:

$$
\lambda_{J}=c_{S} \sqrt{\frac{\pi}{G \rho_{0}}}
$$

where $c_{s}$ is the adiabatic sound speed, and $\rho_{0}$ is the density of the unperturbed selfgravitating liquid. he Jeans mass $M_{i}$ is the mass contained in a Jeans sphere of radius $R_{J}=\frac{1}{2} \lambda_{J}$. Note, that the traditional approach to the Jeans insability implies isentropic (adiabatic) flows, i.e. $S=$ const where $S$ is entropy of the self-gravitating liquid [9-14].

In our treatmant to the Jeans instablity we adopt the simple qualitative appoach. Consider the isothermic ( $T=$ const) spherical homogeneous self-gravitating cloud built of the identical particles, and assume the constant initial density of the cloud $\rho_{0}=$ const. Let us see the cloud as an ideal gas. Two opposing physical factors control the behabior of the cloud. The gas pressure, caused by the thermal movement of the particles comprising the cloud, tries to expand the cloud, whereas gravitation forces the cloud to collapse. The balance of these factors controls the physical behavior of the cloud. Compression the cloud to a radius $r-d r$ demands work $d A$ done against the gas pressure, given by Eq. 3:

$$
d A=-p_{e n} d V=-p_{e n} 4 \pi r^{2} d r
$$

where $p_{e n}$ is the "entropy pressure". Labeling of the pressure as the "entropy one" emphasizes the assumption implying that our self-gravitating cloud behaves as an ideal gas. Recall, that the pressure emerging under isothermic compression of an ideal gas is of pure entropic nature: indeed, the internal energy of the ideal gas under isothermic conditions remains unchanged [16]. The entropic pressure is calculated according to Eq. 4: 


$$
p_{e n}=\frac{F_{e n}}{4 \pi r^{2}}=\frac{1}{4 \pi r^{2}} T \frac{d S}{d r}
$$

where $F_{\text {en }}=T \frac{d S}{d r}$ is the entropic force $[16,17], T$ and $S$ are the temperature and entropy of the cloud respectively. During the compression, the gravitational energy is released. When this energy equals the amount of work to be done on the gas, the critical (Jeans) mass is attained. Equating the work to the change in the gravitational energy yields:

$$
\begin{gathered}
d A=d U_{g r} \\
T \frac{d S}{d r}=\frac{G M^{2}}{2 r^{2}}
\end{gathered}
$$

Considering $M=\rho_{0} \frac{4}{3} \pi r^{3}$ and substitution into Eq. 6 gives rise to Eq. 7:

$$
T \frac{d S}{d r}=\frac{8 \pi^{2}}{9} G \rho_{0}^{2} r^{4}
$$

Integration of Eq. 7 results in Eq. 8:

$$
S(r)=\frac{8 \pi^{2}}{45} G \rho_{0}^{2} \frac{r^{5}}{T}+S_{0}
$$

where $S_{0}$ is the constant of integration, to be established from the third law of thermodynamic. Denoting the Jeans entropy $\Delta S_{J}=S(r)-S_{0}$, considering $M=\rho_{0} \frac{4}{3} \pi r^{3}$ and involving Eq. 1 results in the scaling relations supplied by Eqs. 9-10:

$$
\begin{aligned}
& \Delta S_{J} \sim \frac{G M^{2}}{T r} \\
& \Delta S_{J} \sim \frac{\Delta U_{g r}}{T}
\end{aligned}
$$

Taking along carefully the constants yields the exact equations:

$$
\begin{aligned}
& \Delta S_{J}=\frac{G M^{2}}{10 T r} \\
& \Delta S_{J}=\frac{\Delta U_{g r}}{5 T}
\end{aligned}
$$

Consider two equilibrium spherical clouds $M, r$ contacting one another (their centers are separated by the distance $2 r$ ). The total Jeans entropy of the clouds is $\Delta S_{J}=\frac{G M^{2}}{5 T r}$. The gravitational force acting between clouds equals: $f_{g r}=\frac{G M^{2}}{4 r^{2}}$; thus, Eq. 11 is easily re-written as:

$$
f_{g r}=-\frac{5}{4} T \frac{\partial S_{J}}{\partial r}
$$

Equation 13, which is within an accuracy of a numerical coefficient (which is close to unity) is an equation describing the temperature-dependent entropic forces [16,17]. We already adopted the "ideal gas model" for our self-gravitating cloud; thus, the Jeans entropy may be identified with the Sackur-Tetrode entropy of the ideal gas [18]:

$$
\Delta S_{J}=k_{B} N \ln \left(\frac{V}{N \lambda_{t h}^{3}}\right)+\frac{5}{2}
$$

where $k_{B}$ is the Boltzmann constant, $N$ is the total number of particles in the cloud, $V$ the volume occupied by the cloud, and $\lambda_{t h}$ is the thermal wavelength, which for the ideal gas is supplied by Eq. 15 (see ref. 18): 


$$
\lambda_{t h}=\sqrt{\frac{2 \pi \hbar^{2}}{m k_{B} T}},
$$

where $m$ is the mass of the particle and $\hbar$ is the Planck constant. In the classical limit $\frac{\boldsymbol{V}}{\boldsymbol{N} \lambda_{\text {th }}^{3}} \gg \mathbf{1}$ takes place; thus, $\boldsymbol{\Delta} \boldsymbol{S}_{\boldsymbol{J}} \cong \boldsymbol{k}_{\boldsymbol{B}} \boldsymbol{N} \boldsymbol{l n}\left(\frac{\boldsymbol{V}}{N \lambda_{\text {th }}^{3}}\right)$ is adopted. Equating this expression to Eq. 12 yields for the thermal length of the self-gravitating cloud, seen as an ideal gas, the following expression:

$$
\lambda_{t h}=n^{-\frac{1}{3}} \exp \left(-\frac{\Delta U_{g r}}{5 N k_{B} T}\right)
$$

where $\boldsymbol{n}=\frac{\boldsymbol{N}}{\boldsymbol{V}}$ is the concentration of particles in the cloud. Quite expectedly, the Boltzmann factor relating the total gravitational energy to the thermal one appears in Eq. 16.

\section{Discussion}

Analysis of the Jeans instability in the isothermal self-gravitating clouds, seen as an ideal gas shows that the equilibrium Jeans entropy of the cloud scales as $\Delta S_{J} \sim \frac{\Delta U_{g r}}{T}$ where $\Delta U_{g r}$ is the total self-gravitational energy of the cloud. This expression hints to the entropy origin of the gravity. The analysis of the Jeans instability enables to eliminate the "holographic principle" involved for the grounding of the entropy origin of the gravitational force. The gravity force acting between two isothermal equilibrium clouds scales in turn as $f_{g r} \sim-T \frac{\partial S_{J}}{\partial r}$, which is typical for entropic forces $[16,17]$. Thus, the analysis of the Jeans instability supports the "entropic origin" of gravity.

\section{Conclusions}

The novel approach to gravity, seen as an emerging force appearing as a consequence of the information associated with the positions of material bodies revolutionized the foundations of physics [4]. This idea suggested by Eric Verlind exploits explicitly the "holographic principle", implying that the description of a volume of space can be thought of as encoded on a lower-dimensional boundary to the region (labeled as the "holographic screen") [19]. The analysis performed by Verlinde and developed by other investigators [1-5] demonstrated that gravity is actually a kind of "entropic phenomena", i.e. forces resulting from the entire system's statistical tendency to increase its entropy, rather than from a particular underlying fields/interactions acting between physical objects. This idea was criticized in refs. 6-8 and it is now subjected to intensive and fruitful scientific discussion. We suggest an alternative approach to the problem, based on the analysis of the Jeans gravitational instability. Jeans instability in self-gravitating clouds appears as an interplay of two opposite tendencies, namely: gravity induced compression and thermal expansion due to the random motion of the particles constituting the cloud. We considered isothermal self-gravitating clouds. In the isothermal clouds (whatever is their physical nature) the thermal pressure emerges as a pure entropic phenomenon; indeed, the internal energy of the isothermal cloud remains constant [16]. Thus, mechanical equilibrium of the cloud is seen a balance of the gravity-induced compression and entropy-driven expansion. We introduced the notion of the "Jeans entropy" denoted $\boldsymbol{\Delta} \boldsymbol{S}_{\boldsymbol{J}}$, i.e. the entropy of the isothermal self-gravitating cloud in thermal and mechanical equilibrium. The analysis of the equilibrium shows that the Jeans entropy of the cloud scales as $\Delta \boldsymbol{S}_{\boldsymbol{J}} \sim \frac{\Delta \boldsymbol{U}_{g r}}{\boldsymbol{T}}$ where $\Delta \boldsymbol{U}_{\boldsymbol{g r}}$ is the total self-gravitational energy of the cloud. This expression supports the "entropic origin" of gravity; however, our analysis enables elimination of the "holographic principle" [19]. We also consider gravity interaction between two isothermal equilibrium isothermal clouds; the Newton gravity force scales in this case as $\boldsymbol{f}_{\boldsymbol{g r}} \sim-\boldsymbol{T} \frac{\boldsymbol{\partial} \boldsymbol{s}_{\boldsymbol{J}}}{\boldsymbol{\partial} \boldsymbol{r}}$, which is typical for entropic forces. We conclude that the analysis of the Jeans instability sheds light 
on the entropic nature of the gravity force, at least in the isothermal situation, addressed in ref. 7 .

Author Contributions: Conceptualization, E.B.; methodology, E.B.; investigation, E.B. writingoriginal draft preparation, E. B.; writing-review and editing, E. B.

All authors have read and agreed to the published version of the manuscript.

Funding: This research received no external funding

Data Availability Statement: The data used to support the findings of this study are available from the corresponding author upon request.

Acknowledgments: The author is thankful to Mrs. Yelena Bormashenko for her kind help in preparing this paper.

Conflicts of Interest: The authors declare no conflict of interest.

\section{References}

1. Verlinde E, On the origin of gravity and the laws of Newton, J. High Energ. Phys. 2011, $29,2011$.

2. Cai, R-G., Cao, L-M., Ohta, N. Friedmann equations from entropic force. Phys. Rev. D 2010, 81, 061501(R).

3. Verlinde, E. Emergent Gravity and the Dark Universe, SciPost Phys. 2017, 2, 016.

4. Visser, M. Conservative entropic forces. J. High Energ. Phys. 2011, 140, 2011.

5. Lee, J. W. On the Origin of Entropic Gravity and Inertia. Found. Phys. 2012, 42, 1153-1164.

6. Kobakhidze, A. Gravity is not an entropic force. Phys. Rev. D 2011, 83, 021502(R).

7. Gao, Sh. Is Gravity an Entropic Force? Entropy 2011, 13(5), 936-948.

8. Yang R. Is Gravity Entropic Force? Entropy 2014, 16(8), 4483-4488.

9. Jeans, J. H.The stability of a spherical nebula, Philos. Trans. R. Soc. Lond. A, 1902, 199, 1-53.

10. Arbuzova, E. V., Dolgov, A. D., Reverberi, L. Jeans instability in classical and modified gravity. Physics Letters $B$ 2014, 39, 279-284.

11. Kremer, G.M., Richarte, M. G., Teston, F. Jeans instability in a universe with dissipation. Phys. Rev. D 2018, $97,023515$.

12. Tsiklauri, D. Jeans Instability of Interstellar Gas Clouds in the Background of Weakly Interacting Massive Particles, Astrophysical J. 1998, 507 (1), 226.

13. Lifshitz, E. M. On the gravitational stability of the expanding universe. Zh. Èksp. Teor. Fiz., 1946, 16, 587-602.

14. Gibbons, G. W. The entropy and the stability of the Universe, Nuclear Physics B, 1987, 292, 784-792.

15. Israelachvili, J. N. Intermolecular and Surface Forces, pp. 61-62, $3^{\text {rd }}$ Ed., Elsevier, Amsterdam. 2011.

16. Bormashenko, Ed. Entropy harvesting, Entropy 2013, 15 (6), 2210-2217.

17. Rubinstein, M.; Colby R. H. Polymer Physics, Oxford University Press, Oxford, UK, 2003.

18. Kittel, Ch. Thermal Physics, J. Wiley and Sons, New York, USA, 1969.

19. Susskind, L. The World as a Hologram, J. Mathematical Physics 1995, 36 (11), 6377-6396. 